\title{
Editorial
}

\section{Parcelación o colectivización: Dilema de la reforma agraria en El Salvador}

En Marzo de 1980 se inició el proceso de reforma agraria, la cual se desarrollarla en tres etapas: la primera fase, impulsada a lravés de los decretos 153 y 154, posibilitó la afectación de propiedades mayores a las 500 hectáreas y la conformación de las cooperativas de producción: la segunda, que suponia afectar las propiedades de 150 hectáreas y más, se modificó a partir de la Constilución Polílica de 1983 y en su esencia fué eliminada; y la lercera, la cual mediante el decrelo 207 permitió el acceso a la tierra a pequefios productores arrendatarios, se desarrolló hasta 1983.

Si bien el proceso de reforma agraria implementado hace ocho anos liene componentes polílicos en cuanto respuesta al desarrollo del movimiento revolucionario, no se puede obviar su importancia en cuanto a cierlos objetivos esperados que son trascendentes en el agro, como por ejemplo: el acceso del campesino a la tierra, la translormación de las relaciones de producción en el campo, la mejora de las condiciones de vida del campesinado, el aumenlo de los niveles de producción, la transformación de los sistemas de circulación y orienlación de la producción según las necesidades de la sociedad. Cierlamente, eslos objetivos son ambiciosos, complejos de lograr y requieren de grandes esfuerzos para alcanzarlos.

No pretendemos evaluar lodo el conjunto de aspectos positivos $\mathrm{y} / \mathrm{o}$ negalivos de la aclual reforma agraria, sino analizar sólo algunos problemas y tendencias que se presentan actualmente y las implicaciones que pueden tener en el futuro desarrollo de la reforma. En este sentido 
nos concentraremos en tres aspectos: el primero a considerar se refiere a los alcances de la reforma agraria en cuanto a la reestructuración de la propiedad de la lierra y el número de benificiarios; el segundo, las tendencias que presenlan el uso del suelo en las cooperativas del sector relormado y las aclitudes de los cooperativistas con respecto al proceso; y el tercero, que se deriva de los dos anteriores y pretende interpretar las propuestas de los distintos partidos políticos en relación a la reforma agraria, asl como las implicaciones que eslas tendrán en el futuro avance o retroceso de dicho proceso.

Con respecto a los alcances de la reforma agraria en relación a la reestructuración de la propiedad de la tierra, podemos señalar que el área agricola incorporada efectivamente a este proceso representa una proporción pequefía del área agrícola nacional. Es precisamente este nivel limitado de ateclación de la tierra, el que nos muestra la debilidad de la reforma agraria en relación al mantenimiento del poder económico tradicional en el agro y a los limitados efectos redistributivos de la propiedad y del ingreso en el sector rural.

Para evidenciar lo anterior es suficiente sefialar que el área reformada (Decretos 154, 842 y 207) es de $334,895.7$ ha, que representa el $15 \%$ del área agrícola nacional. Para 1986/87 las propiedades incorporadas por medio del decrelo 154 constiluían un área de $234,779.7$ ha, de las cuales los beneficiarios sólo habían tenido acceso al $87.9 \%(206,394.3$ $\mathrm{Ha}$ ), debido a la aplicación de los derechos de reserva de los antiguos propielarios y al establecimiento de áreas de reserva nacional.

De esta forma las cooperalivas del sector reformado se han desarrollado en un $11 \%$ de lotal del área agrícola nacional, pero conslituyen el $61.6 \%$ del total del área de la relorma agraria. Las pequenas propiedades parcelarias del decreto 207 constiluyen solamente el $3 \%$ del área agricola nacional y se desarrollan en $71,600.4$ ha. que representan el $21.4 \%$ del área del sector reformado. Por otro lado, las unidades que administradas por el ISTA antes del proceso, las cuales se incorporaron al área reformada por medio del decrelo 842, ocupan un área de 56,901 ha.

En la actualidad hay un total de 322 cooperativas que están funcionando, la mayoria (94.7\%) corresponden a las creadas por el decreto 154 , pero también existen 28 cooperativas que han dejado de operar en un área de 14,309.3 ha, lo cual representa casi el $7 \%$ del total del área de las cooperativas activas. La causa fundamental de que estas cooperativas estén abandonadas es el conflicto amado. También a los antiguos propielarios de las tierras alectadas por el decrelo 154, se les ha devuelto un total de 12,545.7 ha. por concepto de Derecho de Reserva.

Para completar nuestra apreciación de que los alcances de la reforma agraria son límitados sefialaremos que el total de beneficiarios para el 
$1986 / 87$ era de 77,269 de los cuales 30,268 correspondla a las cooperativas y 41,001 a las pequenas propiedades parcelarias del decreto 207. Ciertamente el número de socios en las cooperativas ha ido disminuyendo con respecto al perlodo inicial (1980/81) en el cual eran de 31,183 . Esta tendencia decreciente de los beneficiarios especialmente en el área colectiva de la reforma agraria se debe al conjunto de problemas de organización, funcionamiento, gestión, etc. que han presentado las cooperativas, propiciando asl el abandono por parte de sus beneficiarios. En el úllimo año el ISTA ha implementado un programa deliberado de incorporación de nuevos beneficiarios en las cooperativas, algunos de los cuales es población desplazada.

Para comprender los límiles del proceso de reforma agraria en su incidencia en el mundo rural sefialaremos que una Misión Interagencial de las Naciones Unidas en un inlorme sobre "La pobreza rural en El Salvador: Elementos básicos para un política campesina", conclula que después de seis años de la relorma agraria, "a pesar que esta habla beneficiado al $22 \%$ de la población rural $(95,200$ tamilias) la mayor parte de la población rural continúa viviendo en una situación donde la pobreza y la miseria dominan su vida día a dla. Este sector que comprendla 200,000 familias sin tierra representa el $51 \%$ de las familias rurales, un incremento del $10 \%$ respecio al porcentaje en $1975^{n}$.

La evaluación de esia Misión de las Naciones Unidas indicaba que "no obstanle, la reforma agraria si parecia haber complejizado la estructura social en el campo, pues a la par de las familias sin tierra aparecen 31,500 familias agnupadas en las cooperativas, (formadas al expropiarse las propiedades con extensiones superiores a las $\mathbf{5 0 0}$ ha.), un sector claramente privilegiado en el medio nural."

Ciertamente los alcances del proceso de reforma agraria son aún limitados si tenemos en cuenta que los beneficiarios en el sector cooperativo han ido disminuyendo y que el acceso a la tierra para la mayorla de la población del agro todavía es un mito.

El segundo aspecto que nos interesa analizar es la lendencia que presenla el uso de suelo en las cooperativas del seclor reformado, (es decir el uso colectivo o individual de la tierra), lendencia que se caracteriza por el abandono de las reformas colectivas de producción y por la mayor importancia que cobran las formas individuales de producción; a su vez exisle una tendencia a aumenlar el área agrlcola sin cullivar y la tierra dedicada a pastos.

Mientras que el área dedicada a la producción colectiva ha loo disminuyendo en los últimos tres anos (de 1984/85 a 1986/87) en una proporción de $8.8 \%$, de 69,094 ha. a 63,049 ha. para los respectivos afos, el área dedicada a la producción individual al interior de las cooperalivas 
ha ido aumentando en un $10 \%$ en los últimos dos años (1985/86 a 1986/87), pasando de 22,515 ha a 24,919 ha.

La tendencia hacia la reducción de las formas de producción colectivas en las cooperativas se manifiesla lanlo en la producción de granos básicos como en la de cultivos de exportación. En el caso de los primeros decreció de un promedio de 19,087 ha. entre los años 1980-85 a 11,960 ha. en 1986/87 es decir en un 37.3\%; el cultivo de frijol, el más alectado. decreció en más de $77 \%$ en el mismo periodo considerado. En el caso de los cultivos de exportación, éstos se redujeron en $17.6 \%$ pasando de 49,222 ha. de promedio entre 1980-85 a 40,541 ha. en 1986/87; la producción de algodón es la que más se redujo llegando a presentar una calda de $67.5 \%$.

Esta tendencia que se maniliesta en el proceso de relorma agraria es de suma gravedad ya que de ella se puede derivar políticas tendientes a propiciar la parcelación. No se Irata de negar la lógica y racionalidad del campesino para enfrentar su propia reproducción material a través de los cultivos individuales, pero el abandono de formas de producción colectivas que pueden generar una mayor conciencia de ayuda mulua, cooperación y de solidaridad en la población campesina se estaría subordinando a una supuesta y relativa eficiencia de la pequena producción parcelaria.

Los otros problemas que se presentan en las unidades de producción cooperativas del sector retormado son: por un lado, que el área agricola sin cultivar ha aumentado de 15,977 ha. a 20, 485 ha. entre 1984/85 y $1986 / 87$, es decir un $28.2 \%$ lo cual muestra las dilicultades de funcionamiento, crédito y ubicación de estas unidades de producción en las zonas cońlictivas. Por otro lado, la tierra dedicada a pastos ha aumenlado en un $9.9 \%$ entre los mismos anos, aspecto que el Ministerio de Agricultura y Ganaderla califica como injustificado dado que "la población bovina no ha aumentado".

El significado de eslas tendencias es preocupante, más si se considera que existe una gran cantidad de población rural sin acceso a la tierra y por otro porque demuestra la ineficiencia que existe en el funcionamiento de las unidades, propiciando as las posibilidades de revertir las formas coleclivas de producción en favor de las formas individuales.

Esta tendencia se reluerza con las actitudes de los campesinos asociados en las cooperativas del sector relormado, (segundo perfil de beneticiarios de la primera etapa de la reforma agraria Decrelo 154 MAG. 1988), que si bien en un $88.5 \%$ se consideran beneficiados por el proceso de reforma agraria por que les permitió seguridad de trabajo, mayor acceso a la tierra, mejores ingresos, etc; el $51.2 \%$ del total de beneficiarlos preferlan trabajar la tierra en forma individual y el $47.9 \%$ en forma colectiva. las razones de la preferencia hacia el trabajo individual 
estan en orden a que esle les permile "Irabajar en su beneficio propio, puede disponer de la cosecha, se sienten más independientes, puede utilizar mano de obra familiar, etc".

Así pues estas lendencias en el uso de la tierra y las aclitudes de los campesinos en el área cooperativa crean un ambiente propicio para fortalecer las concepciones individualistas en la producción y el rechazo o abandono de las formas colectivas de producción.

Abordaremos ahora el tercer punto referente a las propuestas que algunos partidos han hecho con respecto a la reforma agraria. El partido Alianza Republicana Nacionalista (ARENA), a través de su candidalo presidencial ha expresado que para lograr "la eficiencia de las unidades productivas del sector reformado esta debe basarse en la entrega de títulos que garanticen la propedad plena e individual"; esta concepción parte del hecho que los campesinos cooperalivisados al no sentirse propietarios de una parcela de tierra no tienen los sulicientes estimulos para Irabajar eficientemente, es importante considerar que si bien existe una lógica de autosubsistencia en los campesinos esto es producto de las condiciones hislórico estructurales y de la forma de organización de la producción a la que el campesino a estado sometido.

Para ARENA el problema fundamental se encuentra en las formas colectivas de producción, para lo cual sugieren que mediante la parcelación de estas tierras y su entrega en propiedad a los distinlos beneficiarios, se generaría una competitividad individual que sería beneficiosa al crecimiento de la producción agrícola; lambién señalan la posibilidad de mantener una parte de las cooperativas en lorma colectiva pero haciendo socios accionarios a los campesinos de la empresa y otra parte de las tierras entregarlas a tílulo individual.

No es de extranar la posición de este partido ya que responde a los intereses de los sectores empresariales y a la tradicional burguesía que concentro la propiedad de la tierra. Lo peligroso de este planteamiento, así como de las lendencias que presenla el uso de la tierra en las cooperativas del sector reformado, es que con la entrega de parcelas individuales en propiedad se estaría propiciando que el campesino se enirente a un medio externo socio-económico adverso en condiciones de debilidad, de tal forma que en el mediano plazo podrían perder sus tierra y ésta volver a concentrarse en aquellos sectores cuyo poder económico y político se pretendia reducir con las medidas relormistas.

Otro de los partidos politicos que se ha manifestado en torno a la reforma agraria es la Democracia Cristiana, que si bien no lo ha expresado en una plataforma programática, los distintos luncionarios del gobierno y las asociaciones campesinas que lo apoyan, han declarado no estar dispuestos a la parcelación de las cooperativas sino a consolidar lo hasla ahora 
ha ido aumenlando en un $10 \%$ en los últimos dos años (1985/86 a 1986/87), pasando de 22,515 ha a 24,919 ha.

La tendencia hacia la reducción de las lormas de producción colectivas en las cooperativas se maniliesta lanto en la producción de granos básicos como en la de cultivos de exportación. En el caso de los primeros decreció de un promedio de 19,087 ha. entre los af́os 1980-85 a 11,960 ha. en 1986/87 es decir en un 37.3\%; el cultivo de Irijol, el más afectado, decreció en más de $77 \%$ en el mismo período considerado. En el caso de los cullivos de exportación, éstos se redujeron en $17.6 \%$ pasando de 49,222 ha. de promedio entre 1980-85 a 40,541 ha. en 1986/87; la producción de algodón es la que más se redujo llegando a presentar una calda de $67.5 \%$.

Esta tendencia que se manifiesta en el proceso de reforma agraria es de suma gravedad ya que de ella se puede derivar políticas tendientes a propiciar la parcelación. No se trata de negar la lógica y racionalidad del campesino para enfrentar su propia reproducción material a través de los cultivos individuales, pero el abandono de formas de producción colectivas que pueden generar una mayor conciencia de ayuda mutua, cooperación y de solidaridad en la población campesina se estaría subordinando a una supuesta y relativa eficiencia de la pequefía producción parcelaria.

Los otros problemas que se presentan en las unidades de producción cooperativas del sector reformado son: por un lado, que el área agrícola sin cultivar ha aumentado de 15,977 ha. a 20,485 ha. entre 1984/85 y $1986 / 87$, es decir un $28.2 \%$ lo cual muestra las dificullades de funcionamiento, crédito y ubicación de eslas unidades de producción en las zonas contlictivas. Por otro lado, la lierra dedicada a pastos ha aumentado en un $9.9 \%$ entre los mismos anos, aspecto que el Ministerio de Agricultura y Ganaderla calilica como injustificado dado que "la población bovina no ha aumentado".

El signiticado de estas tendencias es preocupante, más si se considera que existe una gran cantidad de población rural sin acceso a la tierra y por otro porque demuestra la ineliciencia que existe en el funcionamiento de las unidades, propiciando asl las posibilidades de revertir las formas coleclivas de producción en favor de las formas individuales.

Esta tendencia se refuerza con las actitudes de los campesinos asociados en las cooperativas del sector relormado, (segundo perfil de beneficiarios de la primera etapa de la reforma agraria Decrelo 154 MAG. 1988), que si blen en un $88.5 \%$ se consideran beneficiados por el proceso de reforma agraria por que les permitió seguridad de trabajo, mayor acceso a la lierra, mejores ingresos, etc; el $51.2 \%$ del total de beneficiarios preferian trabajar la lierra en forma individual y el $\mathbf{4 7 . 9 \%}$ en forma colectiva. las razones de la preterencia hacia el trabajo individual 
eslan en orden a que este les permile "Irabajar en su beneficio propio. puede disponer de la cosecha, se sienten más independientes, puede utilizar mano de obra familiar, etc".

Asl pues estas tendencias en el uso de la tierra y las aclitudes de los campesinos en el área cooperativa crean un ambiente propicio para fortalecer las concepciones individualistas en la producción y el rechazo o abandono de las formas colectivas de producción.

Abordaremos ahora el tercer punto referente a las propuestas que algunos partidos han hecho con respecto a la reforma agraria. El partido Alianza Republicana Nacionalista (ARENA), a través de su candidato presidencial ha expresado que para lograr "la eliciencia de las unidades productivas del sector reformado esta debe basarse en la entrega de tílulos que garanticen la propedad plena e individual"; esta concepción parte del hecho que los campesinos cooperativisados al no senlirse propietarios de una parcela de tierra no lienen los suficientes eslímulos para trabajar eficientemente, es importante considerar que si bien existe una lógica de autosubsistencia en los campesinos eslo es produclo de las condiciones histórico estruclurales y de la forma de organización de la producción a la que el campesino a estado sometido.

Para ARENA el problema lundamental se encuentra en las formas colectivas de producción, para lo cual sugieren que medianle la parcelación de estas tierras y su entrega en propiedad a los distintos beneficiarios, se generaria una competitividad individual que seria beneficiosa al crecimiento de la producción agrícola; también señalan la posibilidad de mantener una parte de las cooperativas en forma colectiva pero haciendo socios accionarios a los campesinos de la empresa y otra parte de las tierras entregarlas a título individual.

No es de extranar la posición de este partido ya que responde a los intereses de los sectores empresariales y a la tradicional burguesía que concentro la propiedad de la tierra. Lo peligroso de este planteamiento, así como de las lendencias que presenta el uso de la tierra en las cooperalivas del sector reformado, es que con la enirega de parcelas individuales en propiedad se estaría propiciando que el campesino se enfrente a un medio externo socio-económico adverso en condiciones de debilidad, de tal forma que en el mediano plazo podrian perder sus lierra y ésta volver a concentrarse en aquellos sectores cuyo poder económico y político se pretendía reducir con las medidas reformistas.

Otro de los partidos políticos que se ha manifestado en torno a la relorma agraria es la Democracia Cristiana, que si bien no lo ha expresado en una platalorma programática, los dislintos funcionarios del gobierno y las asociaciones campesinas que lo apoyan, han declarado no estar dispuestos a la parcelación de las cooperativas sino a consolidar lo hasta ahora 
logrado. Sus propuestas van èn la linea de declarar autogestionables las empresas cooperativas relegando asi el papel de las instituciones del gobierno como apoyo al desarrollo de estas empresas, lo cual es el resultado de la evidencia de que la acción del Estado en la gestión lécnicoadministrativa de las cooperativas ha presentado serias deficiencias.

El acceso a la tierra para la mayoría de los campesinos que en la actualidad no la poseen se resolvería según este partido a Iravés de la creación de un banco de tierras la cual se encargarla de comprar y vender voluntariamente, en este sentido se daría por finalizada la reforma agraria y lo único que quedaría serla fortalecerla y modemizarla.

Por último, otra fuerza política como es la Convergencia Democrática que reune al Movimienlo Nacional Revolucionario (MNR), al Movimiento Popular Social Cristiano (MPSC) y al Partido Social Demócrata (PSD), se han manifestado por la profundización de la reforma agraria, especialmente considerando que se debe desarrollar la segunda fase de esta, tal como estaba concebida en sus inicios, es decir afectando las propiedades hasta las 150 ha; lo cual significa que los límites que impone la Conslilución Política a la propiedad de la lierra ( 245 ha.) deberian modificarse. También están en contra de la parcelación de la lierra de las cooperativas, pero consideran la necesidad de buscar formas creativas de organización de la producción que beneficien a los campesinos. Es también necesario senalar que esta fuerza política no precisa la forma en que enfrentará el conjunto de problemas que aclualmente constituye un freno para el desarrollo más efectivo de la reforma agraria.

En general existen diferentes concepciones para enirentar los problemas actuales del agro y del sector reformado, lo que la realidad nos muestra es que este sector se encuentra con serios problemas, los alcances de la reforma agraria no parecen haber beneficiado a la mayoría de la población del agro y las tendencias al abandono de las formas colectivas de producción en el área reformada, parecen poner en peligro el desarrollo de formas de organización que pueden potenciar la solidaridad y el apoyo mutuo de los campesinos para entrentar en mejores condiciones los factores externos adversos a las unidades productivas.

Los problemas de la reforma agraria son diversos, sus aspectos de mala gestión lécnico-administrativa, la falta de organización de los campesinos, poca conciencia cooperativa de trabajo, inadecuadas formas de empleo generadas en las cooperativas, relaciones desiguales con los campesinos sin tierras, insuficiente crédito, ineficiente apoyo inslilucional, deficiente asistencia técnica, poco apoyo a la comercialización, etc. Son algunos de los problemas que hacen necesario establecer una polltica más integral de desarrollo para potenciar la reforma agraria y avanzar sobre un mayor acceso a la tierra a la mayoría de los campesinos. 